\title{
Myocardial stunning by gated SPECT: An old tool reinvented in a stunning turn
}

\author{
Wael A. AlJaroudi, MD, FASNC, and Fadi G. Hage, MD, FASNC \\ a Division of Cardiovascular Medicine, Clemenceau Medical Center, Beirut, Lebanon \\ b Division of Cardiovascular Disease, Department of Medicine, University of Alabama at \\ Birmingham, Birmingham, AL \\ c Section of Cardiology, Birmingham Veterans Affairs Medical Center, Birmingham, AL
}

Received Nov 3, 2017; accepted Nov 3, 2017

doi: 10.1007/s12350-017-1127-1

\section{See related article, pp. 833-840}

Stunning: to deprive of strength; to astonish; to shock or overwhelm; to daze or bewilder.

Dictionary.com

Left ventricular (LV) myocardial stunning is a phenomenon described decades ago as a transient acute systolic dysfunction secondary to myocardial ischemia. ${ }^{1}$ The pathogenesis of myocardial stunning involves disruption of calcium homeostasis and oxygen-free radical injury. $^{2}$ In patients with normal myocardial perfusion, LV myocardial contractility increases during stress testing, whether exercise or pharmacologic, with correspondent increase in LV ejection fraction (EF). ${ }^{3-6}$ This is most evident during stress echocardiography where live stress imaging shows augmented contractility of myocardial segments. In patients with significant coronary artery disease (CAD), supply demand mismatch during stress testing results in myocardial perfusion abnormality followed by abnormal segment contractility, an energy-dependent phenomenon, causing transient stunning of the myocytes. ${ }^{7}$ While this is more common with exercise or dobutamine, vasodilator stress testing can also induce myocardial ischemia (steal-phenomenon). If the ischemic burden is significant, global LVEF may decrease at peak stress in up to half of patients. The best predictor of this post-stress stunning is

Reprint requests: Fadi G. Hage, MD, FASNC, Division of Cardiovascular Disease, Department of Medicine, University of Alabama at Birmingham, 306 Lyons-Harrison Research Building, 701 19th Street South, Birmingham, AL 35294-0007; fadihage@uab.edu J Nucl Cardiol 2019;26:841-4.

$1071-3581 / \$ 34.00$

Copyright (C) 2017 American Society of Nuclear Cardiology. the ischemic burden. ${ }^{3}$ However, it is important to point out that factors other than myocardial ischemia, such as hemodynamics and myocardial innervation, play a key role in the lack of augmentation of LVEF with vasodilator stress. $^{5,6}$

Stress-induced new wall motion abnormality is highly specific for $\mathrm{CAD}$ and ischemia with imaging modalities that focus on myocardial motion such as echocardiography and cardiac magnetic resonance imaging. ${ }^{7}$ With gated Tc-99m single-photon emission computed tomography (SPECT), reversible regional wall motion abnormalities with exercise have a specificity of $100 \%$ to detect significant CAD (>70\% epicardial stenosis of a coronary artery on angiogram), with good correlation with coronary artery jeopardy score $(R=0.49, P<0.0001)$, although with limited sensitivity $(53 \%){ }^{8}$ After adjusting for Duke Treadmill Score and other covariates, reversible wall motion abnormality on stress gated SPECT remains an independent predictor of severe CAD. Similarly, worsening of LVEF with vasodilator 201-Thallium-gated SPECT has a high specificity for significant CAD, albeit with low sensitivity. ${ }^{9}$

In addition to global LVEF, assessment of regional wall motion is also feasible with gated SPECT myocardial perfusion imaging (MPI) although quantification and scoring are not routinely performed and reported. The visual assessment of segment thickening is subjective and sometimes limited by the tracer count, frame rate, and attenuation artifact. Another tool provided by gates images is mechanical synchrony using phase analysis. This valuable tool is automated, reproducible, repeatable, and transcends the 8 to 16 frame rate of the gated images though a complex Fourier transformation giving it an apparent frame rate of 1/64th frames/cycle. ${ }^{10}$ Moreover, phase analysis provides regional synchrony and onset of mechanical contractility for each myocardial segment. During stress testing, the 
improvement in systolic function and contractility reserve translates into improvement in mechanical synchrony and narrower phase SD and histogram bandwidth. ${ }^{4}$ The effect of ischemic burden on mechanical synchrony is controversial. In a small study, the presence of reversible perfusion defect did not alter the indices of mechanical dyssynchrony by phase analysis from gated SPECT; however, there was no angiographic data to correlate with anatomic stenosis. ${ }^{11}$ In a more recent study, patients with multi-vessel CAD confirmed with angiography had significantly more global and territorial dyssynchrony at early post-stress than at rest 201-Thallium SPECT MPI. ${ }^{12}$ Another study measured LV synchrony immediately after reperfusion therapy using real-time three-dimensional echocardiography and showed strong correlation with 201-Thallium and 123Ibeta methyl-iodophenyl pentadecanoic acid mismatch with dual isotope SPECT, a reflection of acute myocardial stunning. ${ }^{13}$ In patients with ischemic cardiomyopathy, independent predictors of worsening LV mechanical dyssynchrony at peak stress were perfusion defect size, resting heart rate, lower LVEF, and lack of contractility reserve. ${ }^{14}$ Furthermore, worsening of mechanical dyssynchrony was an independent predictor of all-cause mortality (HR 1.19 [1.01;1.38], per $10^{\circ}$ increase in phase $\mathrm{SD}, P=.04)$ and reclassified $18 \%$ of patients with IDI $1.4 \%(P=.02)$ and net reclassification index of $9 \%(P=.057) .^{14}$

Although most studies examining stunning focused on impaired systolic function and contractility, the diastolic function which is also an energy-dependent process, is affected prior to systolic function. In a recent study, worsening of diastolic function and a change in $E /$ $e^{\prime}>25 \%$ (stress-rest) increased the likelihood of a positive stress echocardiogram, and of obstructive CAD by angiography. ${ }^{15}$ Similarly, diastolic dyssynchrony was shown to be associated with peri-infarct stunned myocardium that is salvaged with primary intervention. ${ }^{16}$

For both systolic and diastolic function assessments with gated MPI, the key parameter is early imaging. While the perfusion images with MPI reflect myocardial perfusion at peak stress (time of tracer injection), segment contractility is a reflection of myocardium at time of imaging which is routinely delayed from peak stress by 15 to 60 minutes depending on type of stress test and tracer. ${ }^{17}$ Stunning is a time-dependent phenomenon that may have resolved by the time-gated images are obtained. In a recent multicenter study from the International Atomic Energy Agency, 229 patients underwent exercise stress SPECT MPI. Stress images were acquired $15 \pm 5$ minutes after radiotracer injection (Stress-1) and repeated at $60 \pm 15$ minutes (Stress-2), while rest images were acquired 60 minutes post injection. The stress LVEF was associated with the degree of ischemia and time of acquisition, and the correlation between ischemic burden and LV stunning was strongest with early imaging (i.e., stress 1). Therefore, the authors concluded that "early post-exercise imaging is feasible, and can potentially improve the detection of post-ischemic stunning without compromising image quality and perfusion data', ${ }^{18}$ In another recent study, Brodov et al ${ }^{19}$ performed serial 2-minutes acquisitions postregadenoson injection with a high-efficiency solid-state CZT SPECT camera in 50 patients. They showed that myocardial stunning (i.e., negative LVEF reserve) was detectable in patients with myocardial ischemia only in early acquisitions (images obtained at the 5th and 9th minutes). Importantly, later acquisitions did not show significant correlation between myocardial ischemia and LVEF reserve. These studies are in line with the robust data demonstrating the prognostic value of LVEF reserve with PET imaging which occurs early after peak stress. $^{5,6,20,21}$

In the current paper, Bestetti et al ${ }^{22}$ evaluated the additional value of systolic wall thickening from gated SPECT images in diagnosing LV myocardial stunning in patients with known significant CAD. Among 2064 patients who underwent 2 days exercise stress/rest 99mTc SPECT MPI (images obtained 45 to 60 minutes after tracer injection), 91 patients were selected for final analysis who had: 1) reversible perfusion defect with summed difference score (SDS) $\geq 5$ and 2) severe CAD ( $\geq 70 \%$ epicardial stenosis) on a coronary angiogram performed within 3 months of the MPI. Wall thickening (WT) was graded on a four-point scale $(0=$ normal, $1=$ mildly impaired, $2=$ moderately impaired, 3 = severely impaired to absent thickening) based on the visual assessment of myocardial wall brightening from diastole to systole using the 20 segment model. WT-SDS was defined as the difference in WT score between stress and rest.

In this cohort with CAD by angiography and ischemic burden on MPI (mean SDS 8.19 \pm 2.9 ), the post-stress LVEF was significantly lower than rest LVEF $\quad(48.1 \% \pm 10.3 \% \quad$ vs. $\quad 50.3 \% \pm 10.7 \%$; $P=0.0001)$, and there was significant segmental wall motion dysfunction with mean WT-SDS 4.44 \pm 4.13 . LV stunning, defined as drop in LVEF (stress-rest) by $\geq 5 \%$, was detectable in $28 \%$ of patients. The study demonstrated an inverse linear correlation between WTSDS and $\triangle \mathrm{LVEF}(\mathrm{RHO}=-0.33, P=0.002)$. Based on the regression equation, a threshold of 11.8 for WTSDS has to be present before a significant drop ( $\geq 5 \%$ ) in LVEF is observed. Furthermore, when the population was divided into those with mild $(\operatorname{SDS}<8)$ and severe ischemia (SDS $\geq 8$ ), WT-SDS was significantly higher in those with severe ischemia $(5.67 \pm 4.11$ vs $3.18 \pm 3.8 ; P=0.003$ ) while $\triangle \mathrm{LVEF}$ was not different 
between the two groups. The authors concluded that WT-SDS quantifies the stunning phenomenon and is an independent parameter with the strongest correlation with SDS and reversible systolic dysfunction ( $\triangle \mathrm{LVEF})$, and hence, should be routinely evaluated and reported.

While the study underscores an important tool to assess the stunning phenomenon that is readily available on gated images but seemingly cast away and ignored, there are several key issues to consider. First, WT is visually assessed and hence affected by readers' expertise; the interobserver and intraobserver variability of the WT-SDS score are not reported. Second, areas of infarct can be tethered by adjacent normal segment contractility, leading to false labeling a segment as having normal contractility and reducing WT-SDS score. Third, the WT-SDS score is a global score that does not reflect regional systolic dysfunction; therefore, WT-SDS will be unchanged if a segment becomes akinetic, while WT in other segments improves during stress. Fourth, the temporal resolution of the gated images with 8 frames/cycle is a limiting effect. Fifth, the value of WT-SDS in vasodilator stress testing needs to be established since this study included only patients undergoing exercise stress. Sixth, the effect of timing of gated image acquisition as discussed above needs to be established. Last, a 20 segment model was used in this study rather than the conventional 17 segment model.

How to define myocardial stunning poses several challenges. If stunning is defined as a drop in LVEF (stress-rest) by $5 \%$, only a quarter of patients with myocardial ischemia in this study had stunning, with the majority of patients (62/91) having no change in LVEF $(\triangle \mathrm{LVEF}$ between $-5 \%$ and $+5 \%$ ). While the authors advocate that WT-SDS is more accurate for defining the stunning phenomenon, WT-SDS ranged from 0 to 15 among those with $\triangle \mathrm{LVEF} \leq-5 \%$. Further, if WTSDS is used to define stunning with a cut-off of 11.8 as the authors established from regression analysis, an even smaller proportion of patients with myocardial ischemia experienced 'myocardial stunning' $(\sim 5 \%)$. The majority of patients even with severe ischemia (SDS $>8)$ had WT-SDS $<10$ as can be seen in Figure $1 \mathrm{~B}$ in Reference ${ }^{22}$ While the ischemic burden is obvious is this cohort, stunning is less so, irrespective of the definition used.

It is important to point out that the prognostic value of WT-SDS is not explored in this study and therefore any use of this marker in lieu of LVEF reserve for risk stratification will need to be examined in future studies. Also, the incremental information provided by some non-perfusion variables may be dependent on the myocardial perfusion pattern, as recently reviewed. ${ }^{23}$ It is of great interest to examine whether WT-SDS or other indicators of myocardial stunning may provide useful information in patients with normal perfusion pattern, but these patients were excluded from this study by design. Lastly, we need to explore whether WT-SDS adds incremental information to other non-perfusion variables such as transient ischemic dilation, ${ }^{24,25}$ STsegment shift, ${ }^{26,27}$ heart rate response, ${ }^{28-30}$ myocardial blood flow reserve, ${ }^{31-33}$ LV dyssynchrony, ${ }^{14,34,35}$ and other non-perfusion variables. ${ }^{23}$

In conclusion, the study by Bestetti et al ${ }^{22}$ reintroduced a long forgotten parameter, WT-SDS, and demonstrated that it correlates better with ischemic burden than LVEF reserve and therefore may improve the detection of myocardial stunning. Larger studies are needed to validate this score, measure its interobserver and intraobserver variability, assess whether regional WT-SDS is better than global score, apply it in cohorts with normal and abnormal myocardial perfusion, and evaluate whether it adds diagnostic and prognostic value beyond LVEF, mechanical dyssynchrony, TID, and other non-perfusion variables. Indeed, we may find out that the information provided may be stunning.

\section{Disclosure}

Dr. Hage has received research Grants from Astellas Pharma USA

\section{References}

1. Opie LH. The ever expanding spectrum of ischemic left ventricular dysfunction. Cardiovasc Drugs Ther 1994;8:297-304.

2. Bolli R, Marban E. Molecular and cellular mechanisms of myocardial stunning. Physiol Rev 1999;79:609-34.

3. Ben-Haim S, Gips S, Merdler A, Front A, Tamir A. Myocardial stunning demonstrated with rest and post-stress measurements of left ventricular function using dual-isotope gated myocardial perfusion SPECT. Nucl Med Commun 2004;25:657-63.

4. AlJaroudi W, Alraies MC, DiFilippo F, Brunken RC, Cerqueira MD, Jaber WA. Effect of stress testing on left ventricular mechanical synchrony by phase analysis of gated positron emission tomography in patients with normal myocardial perfusion. Eur J Nucl Med Mol Imaging 2012;39:665-72.

5. Murthy VL, Dorbala S. Clinical value of hyperemic left ventricular systolic function in vasodilator stress testing. J Nucl Cardiol 2017;24:1002-6.

6. Port S. Interpreting the change in left ventricular ejection fraction during pharmacological coronary vasodilation: Proceed with caution! J Nucl Cardiol 2017;24:998-1001.

7. Sicari R, Cortigiani L. The clinical use of stress echocardiography in ischemic heart disease. Cardiovasc Ultrasound 2017;15:7.

8. Emmett L, Iwanochko RM, Freeman MR, Barolet A, Lee DS, Husain M. Reversible regional wall motion abnormalities on exercise technetium-99m-gated cardiac single photon emission computed tomography predict high-grade angiographic stenoses. J Am Coll Cardiol 2002;39:991-8.

9. Hung GU, Lee KW, Chen CP, Yang KT, Lin WY. Worsening of left ventricular ejection fraction induced by dipyridamole on Tl- 
201 gated myocardial perfusion imaging predicts significant coronary artery disease. J Nucl Cardiol 2006;13:225-32.

10. Chen J, Garcia EV, Bax JJ, Iskandrian AE, Borges-Neto S, Soman P. SPECT myocardial perfusion imaging for the assessment of left ventricular mechanical dyssynchrony. J Nucl Cardiol 2011;18:685-94.

11. Aljaroudi W, Koneru J, Heo J, Iskandrian AE. Impact of ischemia on left ventricular dyssynchrony by phase analysis of gated single photon emission computed tomography myocardial perfusion imaging. J Nucl Cardiol 2011;18:36-42.

12. Huang WS, Huang $\mathrm{CH}$, Lee CL, Chen CP, Hung GU, Chen J. Relation of early post-stress left ventricular dyssynchrony and the extent of angiographic coronary artery disease. J Nucl Cardiol 2014;21:1048-56.

13. Maruyama Y, Masaki N, Shimizu Y, Honda N, Yoshimoto N. Correlation of left ventricular dyssynchrony with myocardial stunning using dual single photon emission computed tomography of (123)iodine-beta-methyl iodophenyl pentadecanoic acid and (201)thallium scintigraphy after reperfusion therapy. Ann Nucl Med 2009;23:799-805.

14. AlJaroudi W, Alraies MC, Menon V, Brunken RC, Cerqueira MD, Jaber WA. Predictors and incremental prognostic value of left ventricular mechanical dyssynchrony response during stress-gated positron emission tomography in patients with ischemic cardiomyopathy. J Nucl Cardiol 2012;19:958-69.

15. Mansour MJ, Aljaroudi W, Mroueh A, Hamoui O, Honeine W, Khoury N, et al. Stress-induced worsening of left ventricular diastolic function as a marker of myocardial ischemia. J Cardiovasc Echogr 2017;27:45-51.

16. Turan B, Dasli T, Erkol A, Erden I, Basaran Y. Diastolic dyssynchrony in acute ST segment elevation myocardial infarction and relationship with functional recovery of left ventricle. J Cardiovasc Ultrasound 2016;24:208-14.

17. Itti E, Levy M, Pouillart F, Perez T, Bellorini M, Rosso J, et al. Thallium gated SPECT: relation between immediate post-stress evolution of ejection fraction and severity of perfusion pattern. Nucl Med Commun 2001;22:57-64.

18. Mut F, Giubbini R, Vitola J, Lusa L, Sobic-Saranovic D, Peix A, et al. Detection of post-exercise stunning by early gated SPECT myocardial perfusion imaging: results from the IAEA multi-center study. J Nucl Cardiol 2014;21:1168-76.

19. Brodov Y, Fish M, Rubeaux M, Otaki Y, Gransar H, Lemley M, et al. Quantitation of left ventricular ejection fraction reserve from early gated regadenoson stress Tc-99m high-efficiency SPECT. J Nucl Cardiol 2016;23:1251-61.

20. Dorbala S, Hachamovitch R, Curillova Z, Thomas D, Vangala D, Kwong RY, et al. Incremental prognostic value of gated Rb-82 positron emission tomography myocardial perfusion imaging over clinical variables and rest LVEF. JACC Cardiovasc Imaging 2009;2:846-54.

21. Dorbala S, Vangala D, Sampson U, Limaye A, Kwong R, Di Carli MF. Value of vasodilator left ventricular ejection fraction reserve in evaluating the magnitude of myocardium at risk and the extent of angiographic coronary artery disease: A $82 \mathrm{Rb}$ PET/CT study. J Nucl Med 2007;48:349-58.

22. Bestetti A, Cuko B, Decarli A, Galli A, Lombardi F. Additional value of systolic wall thickening in myocardial stunning evaluated by stress-rest gated perfusion SPECT. J Nucl Cardiol 2017. https://doi.org/10.1007/s12350-017-1115-5.

23. Bajaj NS, Singh S, Farag A, El-Hajj S, Heo J, Iskandrian AE, et al. The prognostic value of non-perfusion variables obtained during vasodilator stress myocardial perfusion imaging. J Nucl Cardiol 2016;23:390-413

24. Lester D, El-Hajj S, Farag AA, Bhambhvani P, Tauxe L, Heo J, et al. Prognostic value of transient ischemic dilation with regadenoson myocardial perfusion imaging. J Nucl Cardiol 2016;23:1147-55.

25. Golzar Y, Olusanya A, Pe N, Dua SG, Golzar J, Gidea C, et al. The significance of automatically measured transient ischemic dilation in identifying severe and extensive coronary artery disease in regadenoson, single-isotope technetium- $99 \mathrm{~m}$ myocardial perfusion SPECT. J Nucl Cardiol 2015;22:526-34

26. Azemi T, Rai M, Parwani P, Baghdasarian S, Kazi F, Ahlberg $\mathrm{AW}$, et al. Electrocardiographic changes during vasodilator SPECT myocardial perfusion imaging: does it affect diagnosis or prognosis? J Nucl Cardiol 2012;19:84-91.

27. Hage FG, Dubovsky EV, Heo J, Iskandrian AE. Outcome of patients with adenosine-induced ST-segment depression but with normal perfusion on tomographic imaging. Am J Cardiol 2006;98:1009-11.

28. Hage FG, Dean P, Iqbal F, Heo J, Iskandrian AE. A blunted heart rate response to regadenoson is an independent prognostic indicator in patients undergoing myocardial perfusion imaging. J Nucl Cardiol 2011;18:1086-94.

29. Andrikopoulou E, Hage FG. Heart rate response to regadenoson: Making the case for its value in clinical practice. J Nucl Cardiol 2016;23:575-80.

30. AlJaroudi W, Campagnoli T, Fughhi I, Wassouf M, Ali A, Doukky R. Prognostic value of heart rate response during regadenoson stress myocardial perfusion imaging in patients with end stage renal disease. J Nucl Cardiol 2016;23:560-9.

31. Dorbala S, Di Carli MF. Cardiac PET perfusion: Prognosis, risk stratification, and clinical management. Semin Nucl Med 2014;44:344-57.

32. deKemp RA, Wells RG, Ruddy TD. SPECT quantification of myocardial blood flow: A journey of a thousand miles begins with a single step (Lao Tzu, Chinese philosopher, 604-531 BC). J Nucl Cardiol 2017. https://doi.org/10.1007/s12350-017-1106-6.

33. Murthy VL, Naya M, Foster CR, Gaber M, Hainer J, Klein J, et al. Association between coronary vascular dysfunction and cardiac mortality in patients with and without diabetes mellitus. Circulation 2012;126:1858-68.

34. Aggarwal H, AlJaroudi WA, Mehta S, Mannon R, Heo J, Iskandrian $\mathrm{AE}$, et al. The prognostic value of left ventricular mechanical dyssynchrony using gated myocardial perfusion imaging in patients with end-stage renal disease. J Nucl Cardiol 2014;21:73946.

35. Hage FG, Aggarwal H, Patel K, Chen J, Jacobson AF, Heo J, et al. The relationship of left ventricular mechanical dyssynchrony and cardiac sympathetic denervation to potential sudden cardiac death events in systolic heart failure. J Nucl Cardiol 2014;21:78-85. 